\title{
Intermediate-risk meningioma: initial outcomes from NRG Oncology RTOG 0539
}

\author{
Leland Rogers, MD, ${ }^{1}$ Peixin Zhang, PhD, ${ }^{2}$ Michael A. Vogelbaum, MD, PhD, ${ }^{3}$ Arie Perry, MD, ${ }^{4}$ \\ Lynn S. Ashby, MD, ${ }^{1}$ Jignesh M. Modi, MD, ${ }^{5}$ Anthony M. Alleman, MD, MPH, ${ }^{6}$ James Galvin, DSc, ${ }^{7}$ \\ David Brachman, MD, ${ }^{1}$ Joseph M. Jenrette, MD, ${ }^{8}$ John De Groot, MD, ${ }^{9}$ Joseph A. Bovi, MD, ${ }^{10}$ \\ Maria Werner-Wasik, MD, ${ }^{8}$ Jonathan P. S. Knisely, MD, ${ }^{11}$ and Minesh P. Mehta, MD ${ }^{12}$
}

1Department of Radiation Oncology, Barrow Neurological Institute, St. Joseph's Hospital and Medical Center, Phoenix, Arizona; ${ }^{2} N R G$ Oncology Statistics and Data Management Center, Philadelphia, Pennsylvania; ${ }^{3}$ Cleveland Clinic Foundation, Cleveland, Ohio; ${ }^{4}$ Department of Pathology, University of California, San Francisco, California; ${ }^{5}$ MidState Medical Center, Hartford HealthCare, Meriden, Connecticut; ${ }^{6}$ Department of Radiological Science, The University of Oklahoma Health Sciences Center, Oklahoma City, Oklahoma; ${ }^{7}$ maging and Radiation Oncology Core QA Group, Thomas Jefferson University, Philadelphia, Pennsylvania; ${ }^{8}$ Department of Radiation Oncology, Medical University of South Carolina, Charleston, South Carolina; ${ }^{9}$ Department of Neuro-Oncology, University of Texas MD Anderson Cancer Center, Houston, Texas; ${ }^{10}$ Department of Radiation Oncology, Medical College of Wisconsin, Milwaukee, Wisconsin; ${ }^{11}$ Department of Radiation Medicine, Hofstra North Shore, Manhasset, New York; and ${ }^{12}$ Miami Cancer Institute Executive Office, Miami, Florida

OBJECTIVE This is the first clinical outcomes report of NRG Oncology RTOG 0539, detailing the primary endpoint, 3-year progression-free survival (PFS), compared with a predefined historical control for intermediate-risk meningioma, and secondarily evaluating overall survival (OS), local failure, and prospectively scored adverse events (AEs).

METHODS NRG Oncology RTOG 0539 was a Phase II clinical trial allocating meningioma patients to 1 of 3 prognostic groups and management strategies according to WHO grade, recurrence status, and resection extent. For the intermediate-risk group (Group 2), eligible patients had either newly diagnosed WHO Grade II meningioma that had been treated with gross-total resection (GTR; Simpson Grades I-III) or recurrent WHO Grade I meningioma with any resection extent. Pathology and imaging were centrally reviewed. Patients were treated with radiation therapy (RT), either intensity modulated (IMRT) or 3D conformal (3DCRT), 54 Gy in 30 fractions. The RT target volume was defined as the tumor bed and any nodular enhancement (e.g., in patients with recurrent WHO Grade I tumors) with a minimum 8-mm and maximum 15-mm margin, depending on tumor location and setup reproducibility of the RT method. The primary endpoint was 3-year PFS. Results were compared with historical controls (3-year PFS: 70\% following GTR alone and $90 \%$ with GTR + RT). AEs were scored using $\mathrm{NCl}$ Common Toxicity Criteria.

RESULTS Fifty-six patients enrolled in the intermediate-risk group, of whom 3 were ineligible and 1 did not receive RT. Of the 52 patients who received protocol therapy, 4 withdrew without a recurrence before 3 years leaving 48 patients evaluable for the primary endpoint, 3-year PFS, which was actuarially $93.8 \%(p=0.0003)$. Within 3 years, 3 patients experienced events affecting PFS: 1 patient with a WHO Grade II tumor died of the disease, 1 patient with a WHO Grade II tumor had disease progression but remained alive, and 1 patient with recurrent WHO Grade I meningioma died of undetermined cause without tumor progression. The 3-year actuarial local failure rate was 4.1\%, and the 3-year OS rate was $96 \%$. After 3 years, progression occurred in 2 additional patients: 1 patient with recurrent WHO Grade I meningioma and 1 patient with WHO Grade II disease; both remain alive. Among 52 evaluable patients who received protocol treatment, 36 (69.2\%) had WHO Grade II tumors and underwent GTR, and 16 (30.8\%) had recurrent WHO Grade I tumors. There was no significant difference in PFS between these subgroups ( $p=0.52$, HR $0.56,95 \% \mathrm{Cl} 0.09-3.35$ ), validating their consolidation. Of the 52 evaluable patients, 44 (84.6\%) received IMRT, and 50 (96.2\%) were treated per protocol or

ABBREVIATIONS AE = adverse event; CGE = cobalt gray equivalent; CNED = continual no evidence of disease; CTV = clinical tumor volume; EBRT = external beam RT; EORTC = European Organisation for Research and Treatment of Cancer; GTR = gross-total resection; GTV = gross tumor volume; IMRT = intensity-modulated RT; MMSE = Mini-Mental State Examination; OAR = organ at risk; OS = overall survival; $\mathrm{PD}=$ progressive disease; PFS = progression-free survival; PRV = planning risk volume; PTV = planning target volume; PTV $\mathrm{PRV}=$ overlap between the PTV and the particular PRV of concern; RT = radiation therapy; RTOG = Radiation Therapy Oncology Group; SD = stable disease; SRS = stereotactic radiosurgery; STR = subtotal resection; WHO = World Health Organization; 3DCRT = 3D conformal RT.

SUBMITTED May 10, 2016. ACCEPTED November 21, 2016.

INCLUDE WHEN CITING Published online October 6, 2017; DOI: 10.3171/2016.11.JNS161170. 
with acceptable variation. AEs (definitely, probably, or possibly related to protocol treatment) were limited to Grade 1 or 2, with no reported Grade 3 events.

CONCLUSIONS This is the first clinical outcomes report from NRG Oncology RTOG 0539. Patients with intermediaterisk meningioma treated with RT had excellent 3-year PFS, with a low rate of local failure and a low risk of AEs. These results support the use of postoperative RT for newly diagnosed gross-totally resected WHO Grade II or recurrent WHO Grade I meningioma irrespective of resection extent. They also document minimal toxicity and high rates of tumor control with IMRT.

Clinical trial registration no.: NCT00895622 (clinicaltrials.gov).

https://thejns.org/doi/abs/10.3171/2016.11.JNS161170

KEY WORDS meningioma; WHO Grade II (atypical); radiotherapy; oncology

$\mathrm{T}$ Reatment of patients with meningioma is most often based on clinical judgment, personal experience, institutional tradition, and retrospective series, all in the absence of Level 1 evidence. Practices have varied, and the establishment of a uniform approach has been hampered not only by the lack of prospective trials, but also, and rather importantly, by inconsistent grading criteria. In recent years, the latter has been addressed by the World Health Organization (WHO) with updated criteria in 2007 and 2016. Previous grading standards were not broadly accepted, but, based on a recently published secondary endpoint analysis of pathology concordance from NRG Oncology RTOG 0539, the WHO 2000 and 2007 standards appear to have been broadly followed, at least among institutions enrolling patients in NRG Oncology RTOG cooperative group trials. ${ }^{40}$

Several cooperative group meningioma protocols have been launched, but they either have met with disappointing results or have failed to reach accrual goals..$^{10,21,25,27}$ The Southwest Oncology Group (SWOG) completed a Phase III trial, with results published in 2015 by Ji and colleagues, assessing mifepristone, an antiprogestin. That study (SWOG-S9005) randomized patients with progressive or recurrent meningioma to receive either oral mifepristone or placebo and showed no improvement in either failure-free or overall survival with mifepristone. ${ }^{21}$

Regarding radiation therapy (RT), prior evidence has been limited to Level IV or V. ${ }^{25}$ Recently, however, 2 Phase II trials, this one from NRG Oncology RTOG and another from the European Organisation for Research and Treatment of Cancer (EORTC 22042-26042), completed accrual, and the data are undergoing analysis. This report describes the initial clinical outcomes from NRG Oncology RTOG 0539, a Phase II trial of observation for low-risk meningioma and of RT for intermediate- and high-risk meningioma. The trial opened in June 2009 and closed ahead of schedule with full accrual in August 2012. The schema and enrollment data are depicted in Fig. 1.

This report focuses on the intermediate-risk cohort (Group 2), composed of patients with newly diagnosed WHO Grade II meningioma treated with gross-total resection (GTR) or recurrent WHO Grade I meningioma, with or without resection of any extent. We address the study's primary endpoint, 3-year progression-free survival (PFS), as well as the mature secondary endpoints of 3-year overall survival (OS) and acute and late adverse events (AEs).

\section{Methods \\ Institutional Review Board Approval, Consent, and Clinical Trial Registration}

This cooperative group protocol was approved by the institutional review boards at each participating study site, and documentation was received at the Radiation Therapy Oncology Group (RTOG, now NRG Oncology) central office. Each patient signed an approved informed consent form before trial enrollment. This protocol (NRG Oncology RTOG 0539) is registered with ClinicalTrials.gov (https://clinicaltrials.gov). The ClinicalTrials.gov identifier is NCT00895622.

\section{Selection Criteria}

Adults 18 years of age or older with a unifocal, histologically documented intracranial meningioma, with no prior history of cranial RT, with Zubrod Performance Status 0-1 and without severe, active comorbidity were eligible for enrollment. Histology, including WHO 2007 tumor grade and subtype, was confirmed for each patient via central pathology review by one of the authors (A.P.). Following central review, patients were partitioned according to specific criteria into 3 groups: Group 1 (low risk), Group 2 (intermediate risk), and Group 3 (high risk), shown in Fig. 1.

\section{Protocol Registration}

Registration took place in 2 steps. Step 1 was initial registration, followed by submission of pathology specimens for central review. Step 2 registration, which occurred after central pathology review, entailed protocol group assignment. After this step, protocol-specified treatment began.

\section{Tumor Grade and Resection Extent}

This report pertains to patients assigned to the intermediate-risk group (Group 2), which includes patients with a newly diagnosed gross-totally resected WHO Grade II meningioma or a recurrent WHO Grade I meningioma irrespective of the resection extent. Resection extent was classified using Simpson criteria ${ }^{43}$ and was based on the neurosurgeons' assessment and postoperative MRI findings, also centrally reviewed (by Bruce Dean-see Acknowledgments - and authors J.M. or A.A.). Gross-totally resected tumors included Simpson Grades I-III.

For patients with a newly diagnosed WHO Grade II 


\section{Phase II Trial of Observation for Low-Risk Meningioma and of Radiotherapy} for Intermediate and High-Risk Meningioma

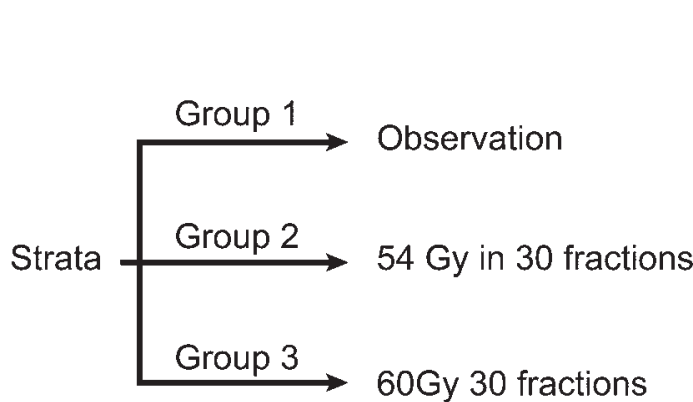

Group 1 (Low Risk):

Group 2 (Intermediate Risk):

Group 3 (High Risk):

FIG. 1. Protocol schema, accrual data, and subgroup definitions.

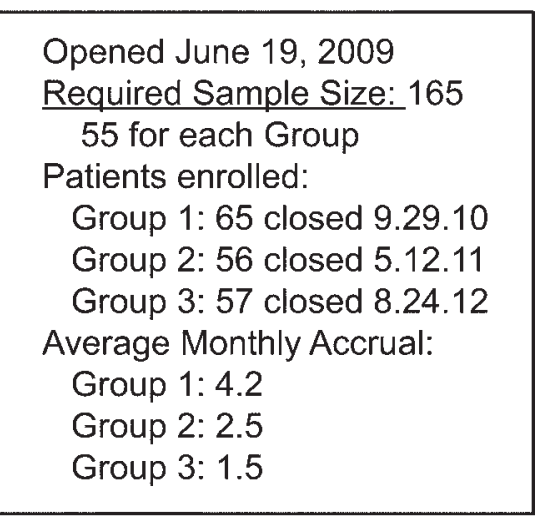

New WHO Grade I, GTR or STR Recurrent Grade I, GTR or STR New WHO Grade II, GTR or STR Any WHO Grade III, GTR or STR Recurrent Grade II, GTR or STR New Grade II, STR meningioma, initial registration and central pathology review must have been completed within 24 weeks of surgery. This interval was designed to permit sufficient time for imaging at 3-4 months after surgery to confirm GTR. In the setting of a recurrent WHO Grade I tumor, there were no such constraints, and although additional resection or biopsy was encouraged, it was not a prerequisite. For patients with WHO Grade I meningioma, recurrence or progression leading to eligibility for protocol enrollment was defined clinically and radiographically at the enrolling institution, with documentary MRI submission as a prerequisite. No patient with a newly diagnosed WHO Grade I meningioma, irrespective of resection grade, was enrolled in Group 2. If further biopsy or resection was performed for recurrent tumor, submission of such specimens was mandated. The diagnosis of recurrence based solely on imaging findings was permitted, but if no additional resection was performed, submission of specimens from the prior resection was required, and tumor grade was centrally confirmed on the basis of those specimens.

Preoperative and postoperative MRI examinations were required for each patient with a WHO Grade II meningioma. For those with recurrent WHO Grade I meningioma, pre- and postoperative MRI examinations were required if surgery was performed; however, only the follow-up imaging documenting recurrence was needed if additional surgery was not undertaken.

\section{Radiation Therapy}

Every Group 2 patient received radiation therapy (RT); 3D conformal RT (3DCRT), intensity-modulated RT (IMRT), or proton therapy was permitted. The dose was $54 \mathrm{~Gy}$ in 30 fractions of $1.8 \mathrm{~Gy}$ each, delivered on consecutive weekdays. The gross tumor volume (GTV) was the tumor or resection bed for all Group 2 patients, plus any nodular enhancement in the recurrent/progressive WHO Grade I subgroup. The GTV was determined on the basis of pre- and postoperative MRI; multiplanar T1-weighted postcontrast and precontrast $\mathrm{T} 1$ - and $\mathrm{T} 2$-weighted and FLAIR images were required. Neither cerebral edema nor the dural tail were included within the GTV, but hyperostotic bone or directly invaded bone was included. The clinical target volume (CTV) was the GTV $+1 \mathrm{~cm}$. It was permissible to reduce the CTV margin to $0.5 \mathrm{~cm}$ around natural barriers to tumor growth such as uninvolved skull. The planning target volume (PTV) was the CTV +3 to $5 \mathrm{~mm}$, depending on the daily RT localization method and reproducibility. A planning risk volume (PRV) was defined for each organ at risk (OAR) - this being the OAR $+3 \mathrm{~mm}$. OAR dose limits were defined in terms of point dose $\left(>0.03 \mathrm{~cm}^{3}\right)$ : lenses $5 \mathrm{~Gy}$, retinae $45 \mathrm{~Gy}$, optic nerves 50 Gy, optic chiasm 54 Gy, and brainstem 55 Gy.

In concept, a PTV accounts for variations in setup and reproducibility. Thus, altering PTV margins to reduce OAR dose is not approved in cooperative group trials. However, in this trial and in the interest of diminishing side-effect risk in cases for which the absolute benefit of RT has not been established with Level 1 evidence, a riskadaptive modification was permitted. If an OAR was in immediate proximity to a PTV such that the dose to the OAR could not be constrained within protocol limits, a

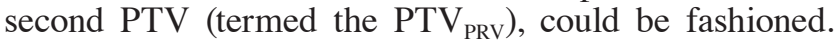

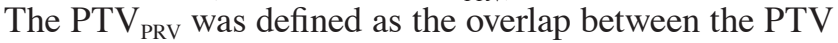
and the particular PRV of concern. If this modification was undertaken, then it was mandated that the dose to the PTV $\mathrm{PRV}_{\mathrm{PR}}$ be as close as permissible to $54 \mathrm{~Gy}$ while not exceeding the OAR dose limit. Figure 2 provides an example of the use of a PTV $\mathrm{PRV}_{\text {. Target volumes and OARs }}$ 


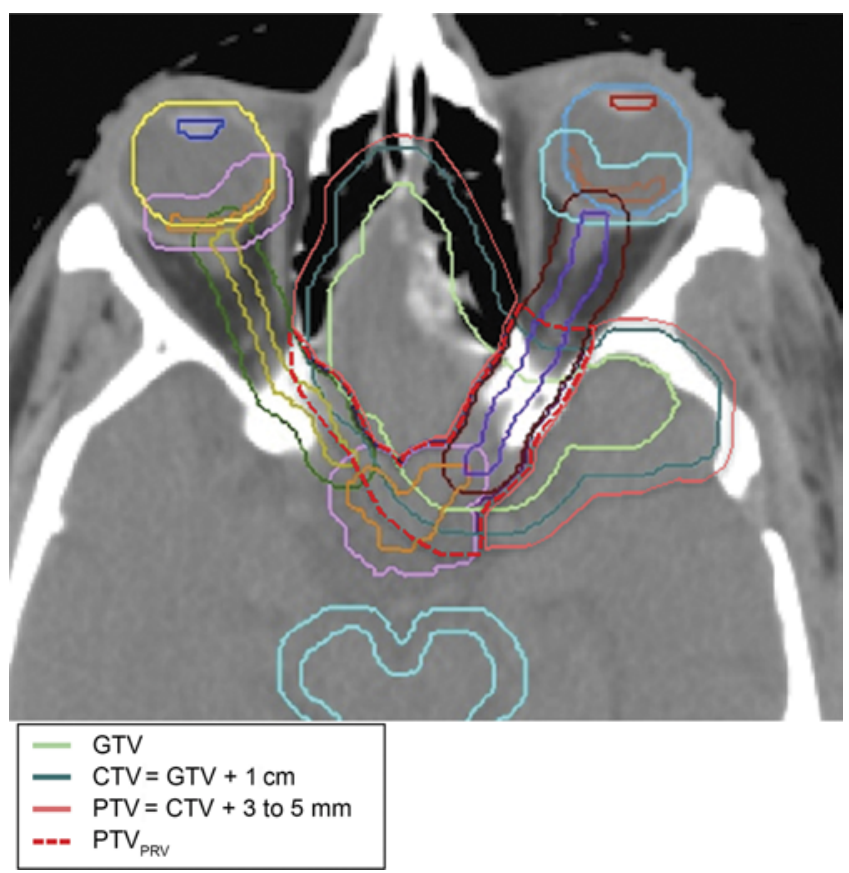

FIG. 2. Planning CT with OAR, PRV, GTV, CTV, PTV, and PTV contours for a patient whose WHO Grade II meningioma surrounded the anterior optic apparatus. The PTV prescription dose was $54 \mathrm{~Gy}$ in 30 fractions. With this example, the PTV $\mathrm{PRV}_{\text {p }}$ prescription was lower to satisfy the optic nerve point dose constraint, while still allowing for the prescribed 54 Gy to the larger PTV.

were reviewed centrally, but this was accomplished after treatment completion.

\section{Patient Assessment}

Pretreatment evaluation included a history and physical with a neurological examination, documentation of steroid use and dosage, documentation of treatment with other hormonal agents, and MRI. Required MRI sequences were multiplanar T1-weighted postcontrast and precontrast T1- and T2-weighted and FLAIR. All patients were required to have had MRI within 12 weeks before Step 2 registration. Both pre- and postoperative MRI studies were required for all newly diagnosed patients. In the setting of recurrent or progressive meningioma without salvage surgery, MRI documentation of recurrence or progression was required. The determination of progression was at the discretion of the enrolling institution. Postoperative MRI studies must have been completed within 12 weeks of surgery, although additional confirmatory imaging was permitted so long as initial registration and central pathology review were completed within 24 weeks of surgery.

A posttreatment clinical assessment was required 1 month after RT, every 3 months for 3 years, and then at least yearly for 10 years. Mini-Mental State Examination (MMSE) and documentation of corticosteroid and other hormonal agent use followed the same schedule. Response was evaluated according to criteria similar to RECIST (Response Evaluation Criteria in Solid Tumors), modified to better apply to meningioma. Continual no evidence of disease (CNED) was ascribed when there was no measurable meningioma, stable disease (SD) when measurable tumor remained unchanged or increased in maximum diameter by less than $20 \%$, and progressive disease (PD) when tumor increased in any diameter by $20 \%$ or more. Neurological progression was defined as new or progressive neurological deficits without measurable growth; this was not observed in any patient. Adverse event evaluations and brain MRI were stipulated at 3 months post-RT, then at least every 6 months for 3 years, then at least yearly for 10 years.

\section{Statistical Methodology}

The primary endpoint of this Phase II trial was PFS at 3 years (3-year PFS) after registration. For this initial evaluation, we report the primary endpoint and secondary endpoints of 3-year OS and AEs. The protocol opened for enrollment on June 19,2009. The full study closed on August 24, 2012, but accrual to the intermediate-risk cohort (Group 2) was completed on May 12, 2011 (see Fig. 1). The analysis date for the present report was January 5, 2016. Findings regarding pathological concordance have been published separately. ${ }^{40}$

Based on historical data, the expected 3-year PFS for this intermediate-risk cohort was estimated at $70 \%$ with GTR alone and at $90 \%$ following GTR + RT. ${ }^{42}$ With a 1 -sided significance level of 0.05 , a sample of 50 eligible patients would provide a statistical power of over $95 \%$ to detect the projected $20 \%$ absolute increase using a 1-sample test on proportion, while providing a greater number of patients for the histopathological and molecular correlative part of the study. The study required the accrual of 55 patients to adjust for a $10 \%$ ineligibility rate.

PFS was measured from the date of study entry to the date of progression or death, or otherwise the date of the last follow-up on which the patient was reported alive and free of disease progression. OS was measured from the date of study entry to the date of death, or otherwise the date of the last follow-up on which the patient was reported alive. Outcomes were estimated using the Kaplan-Meier method. Time to tumor progression was calculated using the cumulative incidence function, with death without progression treated as the competing risk. The incidence rates of Grade 2+ acute and late AEs for dermatological/skin, neurological, and ocular/visual (excluding alopecia) categories, individually and combined, were reported for all eligible patients who received protocol treatment. Acute AEs were defined as AEs that occurred $\leq 90$ days from the start of RT, and late AEs as those that occurred $>90$ days from the start of RT.

We hypothesized that IMRT would minimize treatment-related late toxicities in the dermatological/skin, neurological, and ocular/visual categories compared with 3DCRT. Recognizing that there are no reports of prospectively collected AEs using NCI (National Cancer Institute) Common Toxicity Criteria for meningioma treated with 3DCRT, we tested the hypothesis of reduced toxicities following IMRT by prospectively comparing the late AEs following IMRT in this study with those following 3DCRT in the low-grade glioma patients on NRG Oncology RTOG 0424, which used the same dose and fractionation and similar definitions of treatment volume. Although 3DCRT was allowed for treatment of intermedi- 
ate-risk patients in the present study, it was expected that $80 \%$ to $90 \%$ of the intermediate-risk patients would be treated with IMRT. With 40 IMRT-treated patients, only large differences could be detected with sufficient power. Therefore, a reduction of $10 \%$ or more in the worst overall Grade 2+ AEs would be considered as supporting the hypothesis.

\section{Results}

\section{Patient Characteristics, Protocol Enrollment, and Treatment Delivery}

The study was activated in June 2009, and accrual for the intermediate-risk group was completed in February 2011, 1 year ahead of the projected schedule. Of the 56 patients enrolled, 52 (92.9\%) were eligible and treated with protocol-specified RT (Fig. 3). Pretreatment and tumor characteristics for the eligible patients are listed in Table 1. Of these 52 patients, $36(69.2 \%)$ had undergone GTR for newly diagnosed WHO Grade II meningioma, and 16 $(30.8 \%)$ had a recurrent WHO Grade I tumor. With the RT technologies permitted in this study, 44 patients $(84.6 \%)$ received IMRT, $8(15.4 \%)$ received 3DCRT, and none were treated with proton therapy. This is the first NRG Oncology RTOG trial of brain cancer treatment with protocolspecific IMRT parameters. The majority of the patients were treated per protocol or with acceptable variations. A PTV $_{\text {PRV }}$ was used in 19 patients, principally to limit dose to the optic apparatus. No statistical association was found between protocol adherence or use of the PTV $\mathrm{PRV}_{\text {target }}$ definition option and progression risk.

\section{PFS, OS, and Local Failure}

The median follow-up time for eligible patients still alive was 3.7 years, with a range from 0.4 to 4.9 years. Of the 52 eligible patients who received protocol treatment, 4 $(7.7 \%)$ withdrew less than 3 years after study entry without disease progression. Based on the 48 patients who were evaluable for the primary endpoint, the 3-year PFS rate was $93.8 \%(p=0.0003)$. There was no significant difference in PFS between the intermediate-risk subgroups of WHO Grade II with GTR and WHO Grade I with recurrent/progressive meningioma $(\mathrm{p}=0.52$, HR $0.56,95 \% \mathrm{CI}$ 0.09-3.35). Within 3 years, 3 patients experienced events affecting the PFS: 1 patient with WHO Grade II meningioma died of disease, 1 patient with WHO Grade II meningioma had disease progression and remained alive, and 1 patient with recurrent WHO I meningioma died of undetermined cause without disease progression. Neither median PFS nor median survival time was reached, with a 3-year PFS rate of $93.8 \%$ and a 3-year OS rate of $96.0 \%$. The respective Kaplan-Meier PFS and OS curves are shown in Figs. 4 and 5.

Two patients experienced local failure within 3 years; both had WHO Grade II tumors that were treated with GTR. This corresponds to a 3-year local failure rate of $4.1 \%$. After 3 years, 2 additional patients experienced disease progression; one had a recurrent WHO Grade I meningioma, and the other a newly diagnosed WHO Grade II tumor. Both remain alive as of this writing. The cumulative incidence curve for time to local failure is shown in Fig. 6. Of the 4 patients with progression, 3 were treated with re-resection and systemic therapy; for the remaining patient, no off-protocol therapy was reported following progression.

\section{Adverse Events}

According to the prespecified analysis of treatment-related AEs (dermatological/skin, neurological, and ocular/ visual) definitely, probably, or possibly related to protocol treatment, reported AEs were limited to Grade 1 or 2, and there were no Grade 3 events or higher. In particular, with respect to acute AEs, 5 patients $(10.9 \%)$ experienced Grade 2 AEs and 10 (21.7\%) experienced Grade 1 AEs as the AEs of highest grade. Regarding late AEs with the same reported relationship to protocol treatment, 13 pa-

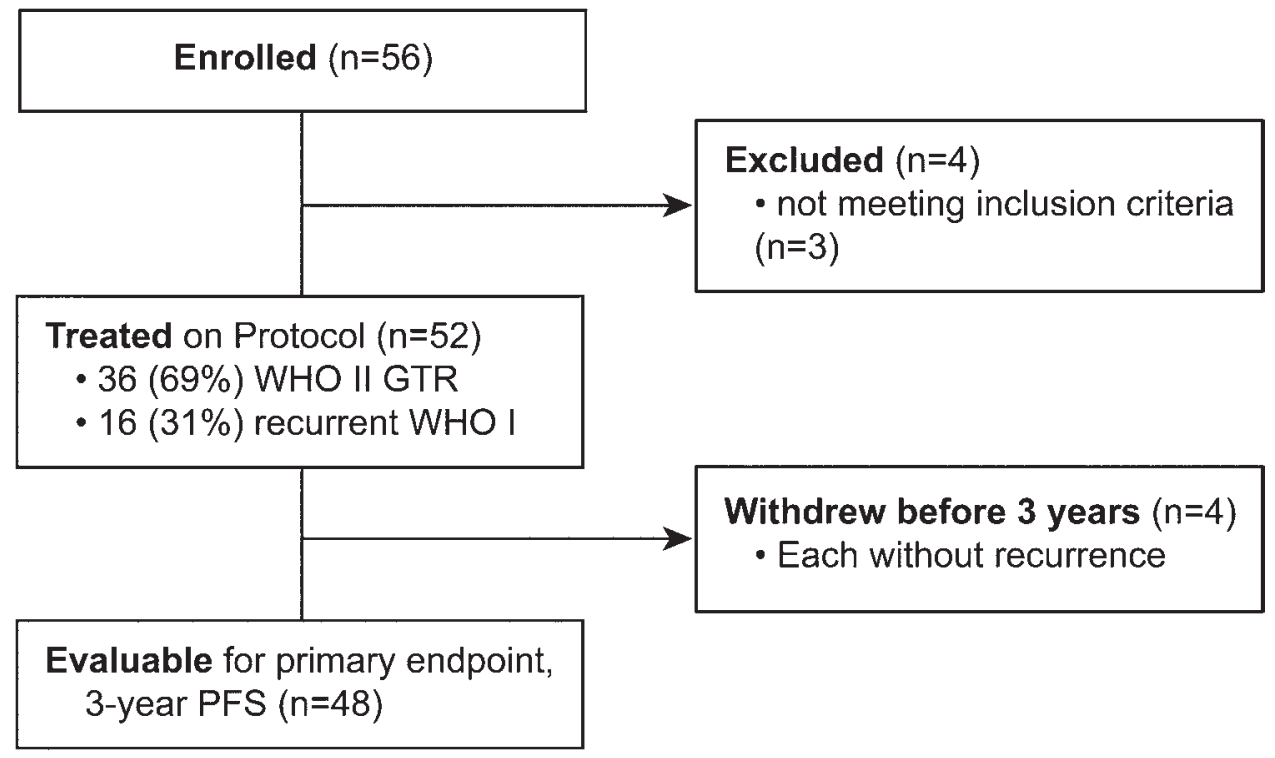

FIG. 3. CONSORT diagram for the intermediate-risk group (Group 2). 
TABLE 1. Patient and tumor characteristics in 52 cases in which patients received the protocol treatment

\begin{tabular}{|c|c|}
\hline Characteristic & Group 2 \\
\hline \multicolumn{2}{|l|}{ Age (years) } \\
\hline$<50$ & $20(38.5)$ \\
\hline$\geq 50$ & $32(61.5)$ \\
\hline \multicolumn{2}{|l|}{ Sex } \\
\hline Male & $20(38.5)$ \\
\hline Female & $32(61.5)$ \\
\hline \multicolumn{2}{|l|}{ Race } \\
\hline American Indian/Alaska Native & $1(1.9)$ \\
\hline Asian & $1(1.9)$ \\
\hline Black or African American & $4(7.7)$ \\
\hline Native Hawaiian or other Pacific Islander & $0(0.0)$ \\
\hline White & $44(84.6)$ \\
\hline Unknown or not reported & $2(3.8)$ \\
\hline \multicolumn{2}{|l|}{ Ethnicity } \\
\hline Hispanic or Latino & $4(7.7)$ \\
\hline Not Hispanic or Latino & $45(86.5)$ \\
\hline Unknown (individuals not reporting ethnicity) & $3(5.8)$ \\
\hline \multicolumn{2}{|l|}{ Pretreatment Zubrod Performance Status } \\
\hline 0 & $39(75.0)$ \\
\hline 1 & $13(25.0)$ \\
\hline \multicolumn{2}{|l|}{ Pretreatment neurological function } \\
\hline No symptoms & $27(51.9)$ \\
\hline Minor symptoms & $21(40.4)$ \\
\hline Moderate symptoms & $4(7.7)$ \\
\hline \multicolumn{2}{|l|}{ Status of tumor } \\
\hline Initial diagnosis & $36(69.2)$ \\
\hline Recurrent & $16(30.8)$ \\
\hline \multicolumn{2}{|l|}{ Extent of resection (Simpson grade) ${ }^{*}$} \\
\hline \multicolumn{2}{|l|}{ At initial surgery } \\
\hline Grade I & $7(13.5)$ \\
\hline Grade II & $17(32.7)$ \\
\hline Grade III & $8(15.4)$ \\
\hline Grade IV & $2(3.8)$ \\
\hline Unknown & $2(3.8)$ \\
\hline \multicolumn{2}{|l|}{ At recurrence } \\
\hline Grade I & $0(0.0)$ \\
\hline Grade II & $0(0.0)$ \\
\hline Grade III & $0(0.0)$ \\
\hline Grade IV & $3(5.8)$ \\
\hline Diagnosis by imaging only & $13(25.0)$ \\
\hline \multicolumn{2}{|l|}{ Histology† } \\
\hline \multicolumn{2}{|l|}{ At initial surgery } \\
\hline WHO Grade I & $2(3.8)$ \\
\hline WHO Grade II & $32(61.5)$ \\
\hline WHO Grade III & $1(1.9)$ \\
\hline Unknown & $1(1.9)$ \\
\hline \multicolumn{2}{|l|}{ At recurrence } \\
\hline WHO Grade I & $2(3.8)$ \\
\hline
\end{tabular}

» CONTINUED FROM PREVIOUS COLUMN

TABLE 1. Patient and tumor characteristics in $\mathbf{5 2}$ cases in which patients received the protocol treatment

\begin{tabular}{cc}
\hline \multicolumn{1}{c}{ Characteristic } & Group 2 \\
\hline Histology† (continued) & \\
\hline At recurrence (continued) & $1(1.9)$ \\
\hline WHO Grade II & $0(0.0)$ \\
\hline WHO Grade III & $13(25.0)$ \\
\hline Identified by imaging only & \\
\hline Lateralization of tumor & $20(38.5)$ \\
\hline Right & $25(48.1)$ \\
\hline Left & $7(13.5)$ \\
\hline Bilateral & $0(0.0)$ \\
\hline Unknown
\end{tabular}

Data are numbers of cases or patients (\%) unless otherwise indicated.

* Simpson resection grades are per enrolling institution.

$\dagger$ WHO grades are per central review.

tients $(25.5 \%)$ and 7 patients $(13.7 \%)$ experienced Grade 2 and Grade 1 AEs, respectively, as the AEs of highest grade. Of the 13 patients with late Grade 2 AEs, 1 was dermatological and 12 were neurological. Some patients with Grade 2 neurological AEs experienced more than 1 AE. The most commonly reported late Grade 2 neurological events were seizure $(n=6)$, speech disorder $(n=3)$, depression $(n=3)$, trigeminal nerve disorder $(n=2)$, olfactory nerve disorder $(n=2)$, peripheral sensory neuropathy $(\mathrm{n}=2)$, memory impairment $(\mathrm{n}=2)$, and dizziness $(\mathrm{n}=2)$. Some patients with Grade 1 late AEs experienced more than $1 \mathrm{AE}$ as well. Seven patients had Grade 1 late AEs, but the total number of AEs they experienced was 15: 2 dermatological, 6 ocular/visual, and 7 neurological. The most common Grade 1 neurological events, in descending order of likelihood, were dizziness, memory impairment, peripheral sensory neuropathy, and peripheral motor neuropathy. The most common ocular/visual events were blurred vision, flashing vision, dry eye, and diplopia.

For AEs with any relationship to protocol treatment, the reported highest-grade AE was Grade 1 in 4 patients (7.7\%), Grade 2 in 35 (67.3\%), and Grade 3 in 8 (15.4\%). There were no reported Grade 4 or 5 events. Among the reported Grade 3 AEs, 3 patients had auditory complaints without categorical evidence of audiometric loss, 2 had neurological complaints, 1 had pain, 1 developed infection, 1 reported skin complaints, and 1 had gastrointestinal symptoms.

Functional outcomes were measured by Zubrod Performance Status, Mini-Mental State Examination (MMSE), and neurological function score. The distributions of these outcomes at baseline, end of RT, and Year 3 are shown in Table 2. For each of these measures, the majority of the patients had either stable or improved status at the end of RT and Year 3.

\section{Intensity-Modulated Radiation Therapy}

We hypothesized that IMRT would minimize late toxicity compared with 3DCRT. Recognizing that there are 


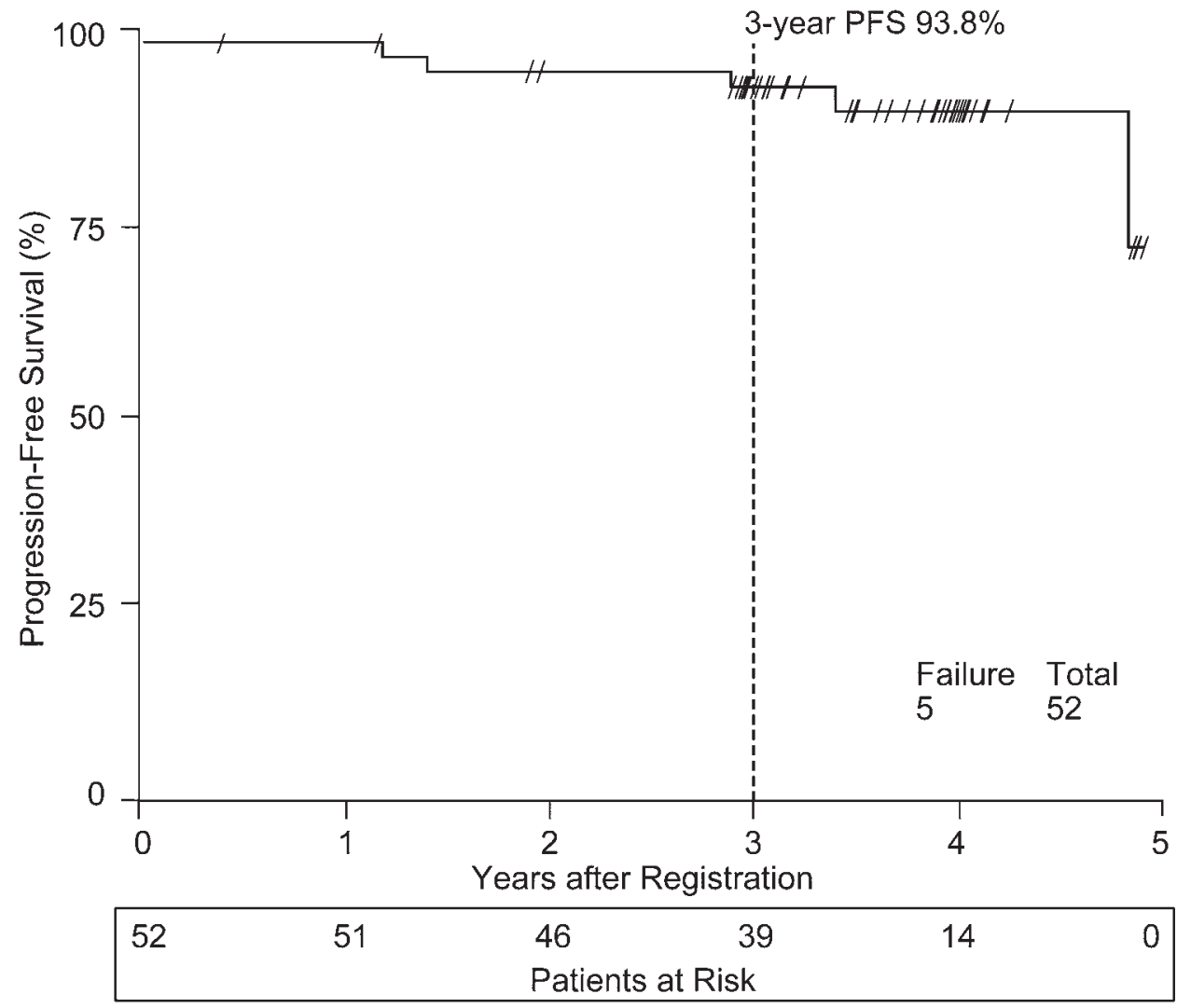

FIG. 4. Progression-free survival, determined with progression and/or death as events. The 3 -year PFS rate was $93.8 \%(95 \% \mathrm{Cl}$ $87.2 \%-100 \%$ ).

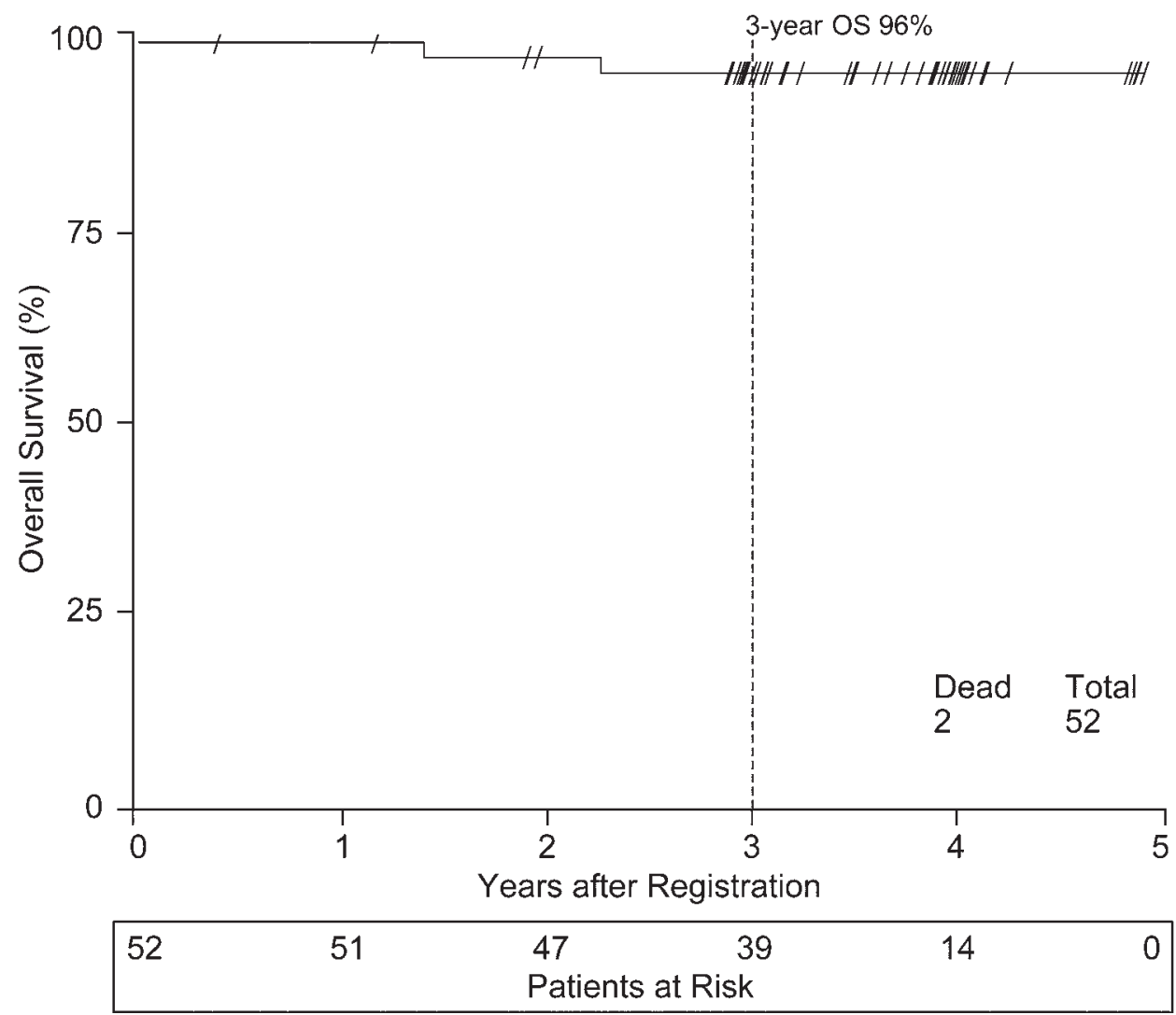

FIG. 5. Overall survival. The 3-year OS rate was $96 \%$ (95\% Cl $90.4 \%-100 \%)$. 


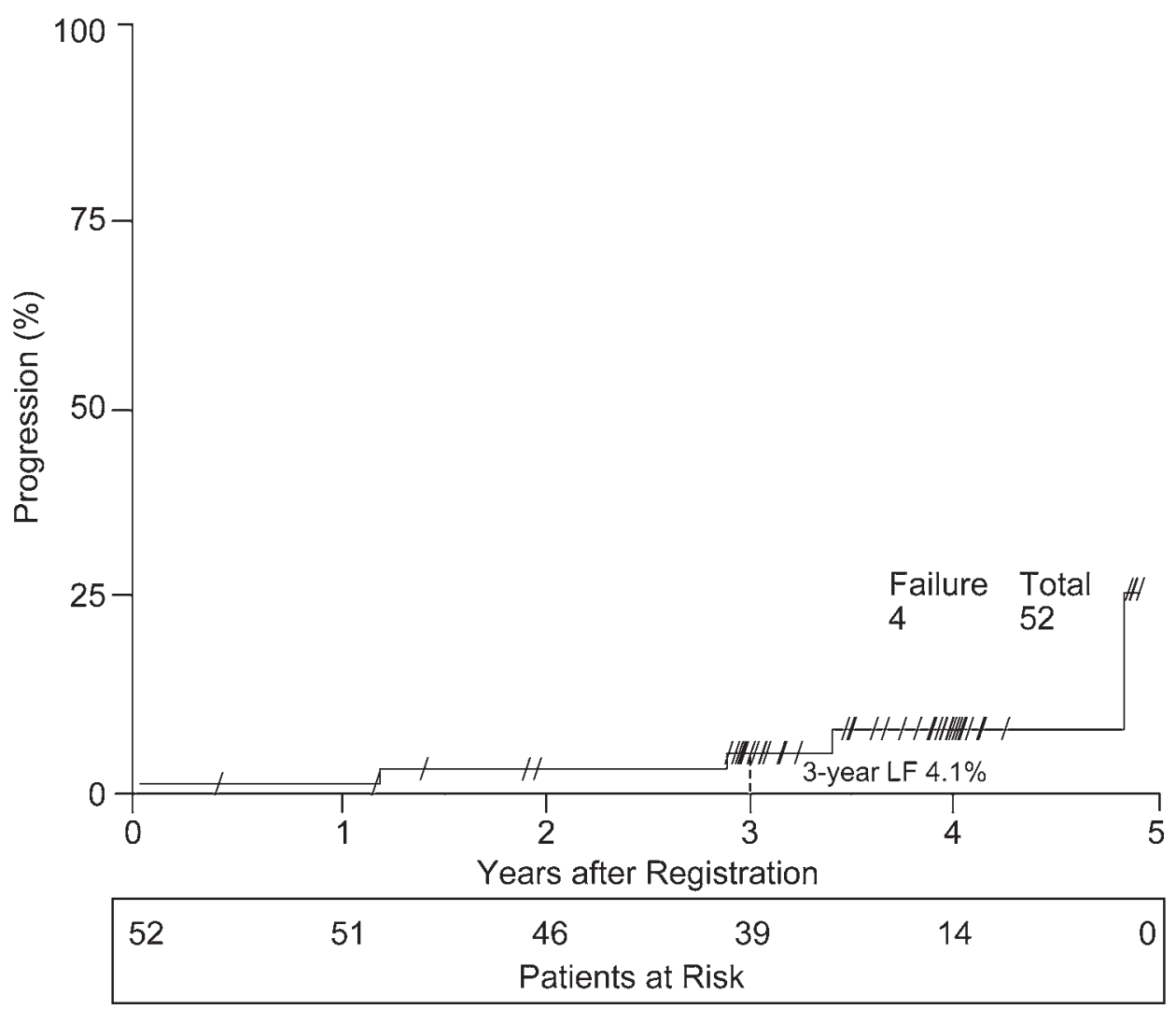

FIG. 6. Local failure. The 3-year local failure (LF) rate was $4.1 \%(95 \% \mathrm{Cl} 0.7 \%-12.5 \%)$.

no reports of prospectively collected AEs using NCI Common Toxicity Criteria for 3DCRT for meningioma, we determined to prospectively test the hypothesis of reduced IMRT toxicity by prospectively comparing late AEs following IMRT on NRG Oncology RTOG 0539 to 3DCRT on NRG Oncology RTOG 0424, a high-risk low-grade glioma study that used the same dose and fractionation and similar definitions of treatment volume. A reduction of $10 \%$ or more in worst overall Grade 2+ AEs was considered supportive. Initial results of the NRG Oncology RTOG 0424 trial have been published, ${ }^{11}$ with additional data supplied by NRG Oncology.

Of the 44 intermediate-risk patients who received IMRT, 43 were evaluable for late AEs. Eleven (25.6\%) of these 43 patients developed Grade 2+ late AEs in the dermatological/skin, neurological, or ocular/visual realms that were deemed definitely, probably, or possibly related to protocol treatment, with 1 patient $(2.3 \%)$ who experienced a Grade $2 \mathrm{AE}$ in the dermatological/skin realm and 10 patients $(23.3 \%)$ who had Grade 2 AEs in the neurological realm. This showed a $17.4 \%$ reduction in the rate of treatment-related Grade 2+ late AEs in those categories with IMRT (Table 3). According to the statistical design described above, these results are considered supportive of the hypothesis that IMRT minimizes late toxicity.

\section{Discussion}

Meningiomas are the most frequently reported primary intracranial neoplasms, accounting for $36.1 \%$ of intracra- nial tumors, compared with $15.4 \%$ for glioblastomas and $28.4 \%$ for all gliomas..$^{33}$ The identification of meningiomas has been increasing over the last several decades, likely related to improved imaging and an aging population rather than actual changes in incidence?

In older series, approximately $90 \%$ of meningiomas were classified as benign, 5\%-10\% atypical, and less than $5 \%$ anaplastic or malignant. ${ }^{20}$ Even in a recent CBTRUS (Central Brain Tumor Registry of the United States) report, WHO Grade II tumors accounted for only $4.2 \%$ of all newly diagnosed meningiomas. ${ }^{24}$ That report, however, was based on a database query and did not include regrading of pathology specimens. Nevertheless, over its study period of 2004-2010, it did document an annual increase in WHO Grade II meningioma of 3.6\%. With improved adoption of modern WHO standards, an increased incidence of WHO Grade II histology and improved correlations between histopathology and clinical outcomes have been documented. Perry and colleagues updated grading criteria and found that approximately $20 \%-25 \%$ of meningiomas fall into the intermediate prognostic group..$^{38,39}$ These grading parameters were adopted by the WHO for their 2000 criteria.

The 2007 and most recent 2016 WHO criteria have added brain invasion as a criterion for Grade II. With these new criteria, the proportion of meningiomas that are classified as WHO Grade II tumors has increased to approximately $25 \%, 37,47$ even reaching $35 \%$ in a single-institution report by Pearson et al. ${ }^{35}$ and $30 \%$ per Backer-Grøndahl and colleagues. ${ }^{3}$ 
TABLE 2. Functional outcome scores

\begin{tabular}{|c|c|c|c|}
\hline \multirow[b]{2}{*}{ Measure } & \multicolumn{3}{|c|}{ Time Point } \\
\hline & Baseline & End of RT & Year 3 \\
\hline \multicolumn{4}{|l|}{ Neurological function } \\
\hline No. of cases w/ data available & 52 & 52 & 42 \\
\hline No symptoms & $27(51.9 \%)$ & $21(40.4 \%)$ & $22(52.4 \%)$ \\
\hline Minor symptoms & $21(40.4 \%)$ & $26(50.0 \%)$ & $9(21.4 \%)$ \\
\hline Moderate symptoms & $4(7.7 \%)$ & $3(5.8 \%)$ & $2(4.8 \%)$ \\
\hline Unknown & 0 & $2(3.8 \%)$ & $9(21.4 \%)$ \\
\hline \multicolumn{4}{|l|}{ MMSE total score } \\
\hline No. of cases w/ data available & 52 & 48 & 27 \\
\hline Median & 30 & 29 & 30 \\
\hline Range (min-max) & $24-30$ & $23-30$ & $26-30$ \\
\hline IQR & $28-30$ & $28-30$ & $29-30$ \\
\hline \multicolumn{4}{|l|}{ Zubrod Performance Status } \\
\hline No. of cases w/ data available & 52 & 52 & 42 \\
\hline 0 & $39(75.0 \%)$ & $35(67.3 \%)$ & $26(61.9 \%)$ \\
\hline 1 & $13(25.0 \%)$ & $12(23.1 \%)$ & $4(9.5 \%)$ \\
\hline 2 & 0 & $1(1.9 \%)$ & 0 \\
\hline Unknown & 0 & $4(7.7 \%)$ & $12(28.6 \%)$ \\
\hline
\end{tabular}

$I Q R=$ interquartile range.

Histopathological grading is a critical element guiding management decisions for patients with meningioma. Large series have independently confirmed tight associations between WHO 2000/2007 histopathological grade and patient outcomes. ${ }^{8,9,17,31,37}$ A secondary analysis from NRG Oncology RTOG 0539, a comparison of histopathological concordance between the enrolling institution and central review, was recently published. ${ }^{40}$ We found a concordance rate of $87.8 \%$ for WHO Grade II, statistically inferior to the concordance for WHO Grades I and III, for which the rates were, respectively, 93.0\% and 93.6\% ( $p<$ 0.0001 ). Twenty-two cases were reclassified after central review. The most common reclassification was from WHO Grade I at the enrolling institution to WHO Grade II after central review (9 cases), although 8 WHO Grade II cases were reclassified as WHO Grade III, and 2 WHO Grade II cases were reclassified as WHO Grade I. Additionally, 2 cases graded as WHO Grade III by the enrolling institution were reclassified as WHO Grade II. In only 1 case did the reclassification not involve WHO Grade II: a tumor diagnosed as WHO Grade I at the enrolling hospital was identified as WHO Grade III on central review.

These findings indicate that the current meningioma classification system is largely interpretable and congruous among pathologists at typically large institutions such as those accruing to this protocol. This was found to be the case with respect to overall meningioma grade, but subjectivities remain in component elements of grading. For instance, there was only slight agreement on focal papillary and focal clear cell; fair agreement for focal rhabdoid, chordoid, and small cells; and moderate agreement on hypercellularity, macronucleoli, sheeting, diffuse papillary, anaplasia, and mitoses $\geq 4$ per 10 high-power fields. ${ }^{40}$ The number of mitoses is a critical element, as it is the most
TABLE 3. Comparison of CTCAE version 3 Grade 2+ late AEs from IMRT in RTOG 0539 (meningioma) versus 3DCRT in RTOG 0424 (low-grade glioma)

\begin{tabular}{ccc}
\hline Variable & RTOG 0539 (IMRT) & RTOG 0424 (3DCRT) \\
\hline No. of patients & 43 & 52 \\
\hline AEs (\% of patients) & & \\
\hline Grade 2+ & 25.6 & 43 \\
\hline Grade 2 & 25.6 & 33 \\
\hline Grade 3 & 0 & 8 \\
\hline Grade 4 & 0 & 2 \\
\hline
\end{tabular}

CTCAE = Common Terminology Criteria for Adverse Events.

common differentiating factor for meningioma grade. Improvements in concordance may require clarifications of the criteria exhibiting lower rates of interobserver concordance and the development of biomarkers predictive of clinical outcome. These are secondary goals of the present trial, awaiting further data maturity and recurrence events.

For patients with WHO Grade I tumors, a Simpson Grade I resection is often curative. Control rates drop somewhat for Simpson Grades II and III, although resections of Simpson Grades I-III are often considered gross total and definitive. With sufficient follow-up, however, recurrence many years following a GTR is common. Retrospective studies with prolonged follow-up have shown progression rates of $15 \%-40 \%$ at 10 years ${ }^{44,45}$ and up to $60 \%$ at 15 years. ${ }^{44}$ Rates in this range have been confirmed in more recently published series as well. ${ }^{13,28}$

Recurrent meningiomas of any grade behave more aggressively than initially diagnosed tumors. After first salvage treatment for a WHO Grade I meningioma, considerably higher rates of subsequent progression have been reported, particularly after surgery alone..$^{29,30,36,45,46}$ For recurrent Grade I meningiomas treated with re-resection, a 3 -year local progression risk of $55 \%-60 \%$ is reported. ${ }^{30,46}$ Stafford et al. identified a $25 \%$ 10-year local progression risk after initial diagnosis and essentially the same risk at 2 years (24\%) after first recurrence. ${ }^{45}$ With specific reference to sphenoid wing meningiomas involving the optic apparatus, often treated with subtotal resection (STR), Peele et al. found a mean interval to first recurrence/progression of 4.4 years, but a considerably shorter mean interval of 14 months from first recurrence to subsequent recurrence or progression. ${ }^{36}$ Mehdorn published an experience with 463 patients, noting that first recurrences were found at a mean of 65 months after surgery, whereas second recurrences developed at a mean of 34 months after the first occurence. ${ }^{29}$ This approximates the rates of first recurrence following gross-total resection of a WHO Grade II meningioma.

With such background data, we formulated an intermediate-risk group composed of patients with a newly diagnosed gross-totally resected WHO Grade II meningioma or with a recurrent WHO Grade I tumor irrespective of resection extent. These patients formed Group 2 in the trial and are the subjects of this report. The results we have observed to date support our decision to include patients with WHO Grade II tumors that have been gross-totally resected as well as patients with recurrent WHO Grade I 

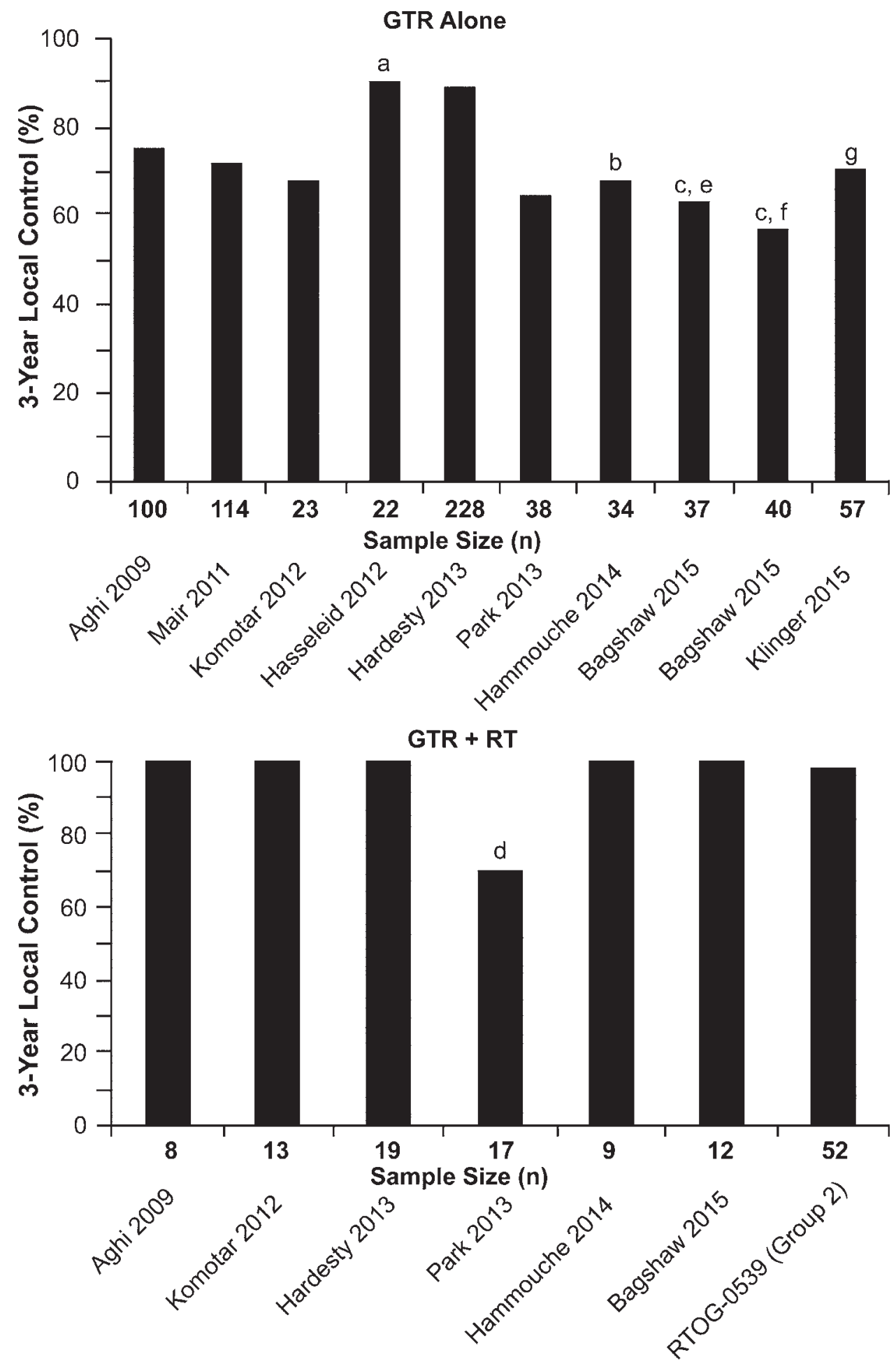

FIG. 7. Three-year local control after GTR alone (upper) versus GTR plus RT (lower). a Convexity meningioma only, 90\% 3-year retreatment-free survival. ${ }^{b}$ Crude 4 -year local control after GTR. ${ }^{c} 63 \%$ for Simpson Grade I resection, $57 \%$ for Simpson Grades IIII. dPre-3DCRT methods used in 18 (67\%) of 27 patients who received RT from 1997 to 2011. eSimpson Grade I. 'Simpson Grades I-III. 'sSimpson Grades I and II; 3 of 57 patients received early adjuvant therapy.

tumors in the intermediate-risk group, albeit with recognition that few recurrent events have occurred in this combined population to date. Moreover, the results of this prospective study corroborate retrospective analyses, which suggest that RT should play a role in the management of intermediate-risk meningioma. Three-year PFS rates from retrospective reports using WHO 2000-2007 grading criteria are shown in Fig. 7:1, 1,14-16,22,23,26,34 included are 10 re- 
ports in which patients were treated with GTR alone and 7 in which they were treated with GTR + RT.

Aghi et al. described 108 patients with atypical meningioma who underwent Simpson Grade I resection, 100 of whom underwent surgery alone and 8 of whom had surgery and EBRT to a mean RT dose of 60.2 Gy. The 5-year recurrence with GTR alone was $45 \%$, but $0 \%$ with surgery and RT $(\mathrm{p}=0.1) .{ }^{1}$ Komotar et al. ${ }^{23}$ reported 45 patients with atypical meningioma and a Simpson Grade I to II resection: $32(71 \%)$ had a GTR alone, and $13(29 \%)$ had a GTR and RT, median RT dose 59.4 Gy. After GTR alone, 13 patients $(41 \%)$ had a recurrence at a median of 19 months. After GTR plus RT, 1 patient $(8 \%)$ had a recurrence at 52.5 months, for respective 6-year actuarial recurrence risks of $65 \%$ versus $20 \%(\mathrm{p}=0.085) .^{23}$

Park and colleagues ${ }^{34}$ analyzed the role of RT following resection of atypical meningioma in 82 patients. Fifty-six were treated with initial surgery alone and 27 with surgery followed by RT. The median dose of RT was $61.2 \mathrm{~Gy}$. Defining GTR as Simpson Grade I to II, they found that postoperative RT significantly improved PFS for the entire cohort, although not for patients with GTR ( $p=0.858)$. Three-year PFS was $65 \%$ after GTR alone and $71 \%$ following GTR + RT, but at 5 years PFS remained $65 \%$ with GTR alone and fell to $53 \%$ after GTR + RT. ${ }^{34}$ This differs from the other recent reports, perhaps owing in part to patient selection, in part to the determination of GTR in their analysis based on the surgeon's report without the requirement for corroborating postoperative imaging, and in part to the fact that many of their patients (18 [67\%] of 27) received RT prior to the incorporation of $3 \mathrm{D}$ image-based treatment planning. Goldsmith and colleagues reported that CT- and MRI-based treatment planning resulted in significantly superior tumor control..$^{12}$

Reports using protons further illuminate outcomes with larger field RT following STR, and provide information that may be important for dose-response assessment for WHO Grade II meningioma. Boskos et al. detailed 24 patients with high-grade meningioma (79\% WHO Grade II), typically following STR. Cause-specific survival at 5 years was $80 \%$ with $>60$ Gy versus $24 \%$ with $\leq 60$ Gy $(p=0.01)$, with a trend toward further improvement with doses $>65$ Gy (p $=0.06) .{ }^{5}$ Hug et al. reported on 15 patients treated with 40 to 72 Cobalt Gray Equivalent (CGE). The 5-year local control rate was $90 \%$ with $>60 \mathrm{CGE}$ and $0 \%$ with $\leq 60 \mathrm{CGE} .{ }^{19}$

Stereotactic radiosurgery (SRS) has become standard in the management of meningioma, and has resulted in favorable outcomes in the primary or adjuvant treatment of WHO Grade I or presumed Grade I tumors. ${ }^{41}$ We did not include SRS as an option with this trial on 4 accounts: 1) patients with newly diagnosed WHO Grade I meningioma were observed even following STR; 2) there was no impetus to include SRS as an option for gross-totally resected WHO Grade II meningioma because no traditional SRS target would be available; 3 ) recurrent WHO Grade I and newly diagnosed gross-totally resected Grade II meningiomas display similar recurrence rates and patterns of progression;32 and 4) SRS for WHO Grade II meningioma has met with high rates of recurrence outside the SRS volume, although in or near (e.g., within $2 \mathrm{~cm}$ of) the resection bed. ${ }^{2,41}$
Huffmann et al. reviewed 15 patients treated with SRS, median dose $16 \mathrm{~Gy}$. At 18 to 36 months, crude local control was $60 \%$. Six (40\%) progressed, 1 in field, but all within the resection bed. ${ }^{18}$ Choi et al. reviewed 25 patients with atypical meningioma, median SRS dose 22 Gy in 1-4 fractions. Recurrence occurred in 9: 3 within the targeted region, 5 elsewhere in the resection bed, and 1 regionally. ${ }^{6}$ These findings suggest that the appropriate target volume for atypical meningioma extends beyond the enhancing tumor, and includes the entire resection bed. Future patterns of failure analyses are needed before definitive guidelines can be developed.

NRG Oncology RTOG 0539 employed a fractionated external beam RT (EBRT) dose of 54 Gy in 30 fractions and found that this was well tolerated, with a favorable AE profile and no serious AEs. This RT dose was chosen over a decade ago, at which time arguments against the use of RT for an intermediate-risk meningioma patient were even more vociferous than at present. However, we now have a larger volume of literature to draw from, suggesting, as described in the aforementioned reports, that higher doses may provide improved PFS.

EORTC 22042-26042 (accrual completed, pending publication), a Phase II trial of postoperative RT for patients with atypical or malignant meningioma, employed 60 Gy following a GTR and added a 10-Gy boost after STR. The definition of GTR as well as target volumes were very similar to those used in the present trial (NRG Oncology RTOG 0539). GTR was defined as Simpson Grade I-III. RT targets included the resection bed with any remaining enhancing tumor, with a $10-\mathrm{mm}$ margin added for subclinical extension, and a planning margin of $5 \mathrm{~mm}$ with conformal or intensity-modulated RT, permitting smaller margins (1-5 mm) if stereotactic methods were employed. This trial will provide further guidance regarding dosing for atypical meningioma, especially once long-term follow-up data are available from both the EORTC and NRG Oncology RTOG trials and comparative outcomes can be assessed.

\section{Conclusions}

NRG Oncology RTOG 0539 has demonstrated that meningioma patients can be successfully enrolled in large cooperative group trials. Indeed, accrual was ahead of schedule. It also shows that patients with intermediaterisk meningioma have limited risk of local failure $(4.1 \%)$ and excellent rates of PFS (93.8\%) and OS (96\%) at 3 years when treated with RT. Comparison with historical control data suggests that 3-year PFS for intermediaterisk patients is better with $\mathrm{RT}$ than with observation. The results of this single-arm study support enrollment to a definitive, Phase III trial evaluating RT versus observation following GTR of WHO Grade II meningioma. In this respect, 2 Phase III trials are presently open to enrollment internationally, NRG BN-003 (http://clinicaltrials. gov/ct2/show/NCT03180268) and the ROAM trial (http:// roam-trial.org.uk).

\section{Acknowledgments}

The late Dr. Bruce Dean, Barrow Neurological Institute, was 
the neuroradiologist instrumental in the trial design. He reviewed all images at protocol inception. It is with gratitude that we acknowledge his contributions.

This project was supported by grants U10CA21661, U10CA180868, and U10CA180822 from the National Cancer Institute to RTOG (now NRG Oncology). In addition, NRG Oncology RTOG 0539 was supported by an ARRA (American Recovery and Reinvestment Act) grant obtained from the NIH by Dr. Rogers.

\section{References}

1. Aghi MK, Carter BS, Cosgrove GR, Ojemann RG, AminHanjani S, Martuza RL, et al: Long-term recurrence rates of atypical meningiomas after gross total resection with or without postoperative adjuvant radiation. Neurosurgery 64:56-60, 2009

2. Attia A, Chan MD, Mott RT, Russell GB, Seif D, Bourland JD, et al: Patterns of failure after treatment of atypical meningioma with Gamma Knife radiosurgery. J Neurooncol 108:179-185, 2012

3. Backer-Grøndahl T, Moen BH, Torp SH: The histopathological spectrum of human meningiomas. Int J Clin Exp Pathol 5:231-242, 2012

4. Bagshaw HP, Jensen RL, Palmer CA, Shrieve DC: Stereotactic radiation therapy and the management of atypical meningiomas: outcomes in the upfront and recurrent setting. Int J Radiat Oncol Biol Phys 93 (3 Suppl):S140, 2015 (Abstract)

5. Boskos C, Feuvret L, Noel G, Habrand JL, Pommier P, Alapetite $\mathrm{C}$, et al: Combined proton and photon conformal radiotherapy for intracranial atypical and malignant meningioma. Int J Radiat Oncol Biol Phys 75:399-406, 2009

6. Choi CY, Soltys SG, Gibbs IC, Harsh GR, Jackson PS, Lieberson RE, et al: Cyberknife stereotactic radiosurgery for treatment of atypical (WHO grade II) cranial meningiomas. Neurosurgery 67:1180-1188, 2010

7. Claus EB, Bondy ML, Schildkraut JM, Wiemels JL, Wrensch M, Black PM: Epidemiology of intracranial meningioma. Neurosurgery 57:1088-1095, 2005

8. Combs SE, Schulz-Ertner D, Debus J, von Deimling A, Hartmann C: Improved correlation of the neuropathologic classification according to adapted world health organization classification and outcome after radiotherapy in patients with atypical and anaplastic meningiomas. Int J Radiat Oncol Biol Phys 81:1415-1421, 2011

9. Domingues PH, Sousa P, Otero Á, Gonçalves JM, Ruiz L, de Oliveira C, et al: Proposal for a new risk stratification classification for meningioma based on patient age, WHO tumor grade, size, localization, and karyotype. Neuro Oncol 16:735-747, 2014

10. EORTC: Radiotherapy or radiosurgery compared with observation alone in treating patients with newly diagnosed, benign meningioma that has been partially removed by surgery. ClinicalTrials.gov. (https://clinicaltrials.gov/ct2/show/ NCT00104936) [Accessed April 20, 2017]

11. Fisher BJ, Hu C, Macdonald DR, Lesser GJ, Coons SW, Brachman DG, et al: Phase 2 study of temozolomide-based chemoradiation therapy for high-risk low-grade gliomas: preliminary results of Radiation Therapy Oncology Group 0424. Int J Radiat Oncol Biol Phys 91:497-504, 2015

12. Goldsmith BJ, Wara WM, Wilson CB, Larson DA: Postoperative irradiation for subtotally resected meningiomas. A retrospective analysis of 140 patients treated from 1967 to 1990. J Neurosurg 80:195-201, 1994

13. Gousias K, Schramm J, Simon M: The Simpson grading revisited: aggressive surgery and its place in modern meningioma management. J Neurosurg 125:551-560, 2016

14. Hammouche S, Clark S, Wong AHL, Eldridge P, Farah JO: Long-term survival analysis of atypical meningiomas: sur- vival rates, prognostic factors, operative and radiotherapy treatment. Acta Neurochir (Wien) 156:1475-1481, 2014

15. Hardesty DA, Wolf AB, Brachman DG, McBride HL, Youssef E, Nakaji P, et al: The impact of adjuvant stereotactic radiosurgery on atypical meningioma recurrence following aggressive microsurgical resection. J Neurosurg 119:475481, 2013

16. Hasseleid BF, Meling TR, Rønning P, Scheie D, Helseth E: Surgery for convexity meningioma: Simpson Grade I resection as the goal: clinical article. J Neurosurg 117:999-1006, 2012

17. Ho DM, Hsu CY, Ting LT, Chiang H: Histopathology and MIB-1 labeling index predicted recurrence of meningiomas: a proposal of diagnostic criteria for patients with atypical meningioma. Cancer 94:1538-1547, 2002

18. Huffmann BC, Reinacher PC, Gilsbach JM: Gamma Knife surgery for atypical meningiomas. J Neurosurg 102 Suppl:283-285, 2005

19. Hug EB, Devries A, Thornton AF, Munzenride JE, Pardo FS, Hedley-Whyte ET, et al: Management of atypical and malignant meningiomas: role of high-dose, 3D-conformal radiation therapy. J Neurooncol 48:151-160, 2000

20. Jääskeläinen J, Haltia M, Servo A: Atypical and anaplastic meningiomas: radiology, surgery, radiotherapy, and outcome. Surg Neurol 25:233-242, 1986

21. Ji Y, Rankin C, Grunberg S, Sherrod AE, Ahmadi J, Townsend JJ, et al: Double-blind phase III randomized trial of the antiprogestin agent mifepristone in the treatment of unresectable meningioma: SWOG S9005. J Clin Oncol 33:4093-4098, 2015

22. Klinger DR, Flores BC, Lewis JJ, Hatanpaa K, Choe K, Mickey B, et al: Atypical meningiomas: recurrence, reoperation, and radiotherapy. World Neurosurg 84:839-845, 2015

23. Komotar RJ, Iorgulescu JB, Raper DMS, Holland EC, Beal $\mathrm{K}$, Bilsky $\mathrm{MH}$, et al: The role of radiotherapy following gross-total resection of atypical meningiomas. J Neurosurg 117:679-686, 2012

24. Kshettry VR, Ostrom QT, Kruchko C, Al-Mefty O, Barnett GH, Barnholtz-Sloan JS: Descriptive epidemiology of World Health Organization Grades II and III intracranial meningiomas in the United States. Neuro Oncol 17:1166-1173, 2015

25. Maclean J, Fersht N, Short S: Controversies in radiotherapy for meningioma. Clin Oncol (R Coll Radiol) 26:51-64, 2014

26. Mair R, Morris K, Scott I, Carroll TA: Radiotherapy for atypical meningiomas. J Neurosurg 115:811-819, 2011

27. Mazza E, Brandes A, Zanon S, Eoli M, Lombardi G, Faedi $M$, et al: Hydroxyurea with or without imatinib in the treatment of recurrent or progressive meningiomas: a randomized phase II trial by Gruppo Italiano Cooperativo di Neuro-Oncologia (GICNO). Cancer Chemother Pharmacol 77:115120,2016

28. McGovern SL, Aldape KD, Munsell MF, Mahajan A, DeMonte F, Woo SY: A comparison of World Health Organization tumor grades at recurrence in patients with non-skull base and skull base meningiomas. J Neurosurg 112:925933, 2010

29. Mehdorn HM: Intracranial meningiomas: a 30-year experience and literature review. Adv Tech Stand Neurosurg 43:139-184, 2016

30. Miralbell R, Linggood RM, de la Monte S, Convery K, Munzenrider JE, Mirimanoff RO: The role of radiotherapy in the treatment of subtotally resected benign meningiomas. J Neurooncol 13:157-164, 1992

31. Olar A, Wani KM, Sulman EP, Mansouri A, Zadeh G, Wilson CD, et al: Mitotic index is an independent predictor of recurrence-free survival in meningioma. Brain Pathol 25:266-275, 2015

32. Onodera S, Aoyama H, Katoh N, Taguchi H, Yasuda K, Yoshida D, et al: Long-term outcomes of fractionated stereo- 
tactic radiotherapy for intracranial skull base benign meningiomas in single institution. Jpn J Clin Oncol 41:462-468, 2011

33. Ostrom QT, Gittleman H, Liao P, Rouse C, Chen Y, Dowling $\mathrm{J}$, et al: CBTRUS statistical report: primary brain and central nervous system tumors diagnosed in the United States in 2007-2011. Neuro Oncol 16 (Suppl 4):ivl-iv63, 2014

34. Park HJ, Kang HC, Kim IH, Park SH, Kim DG, Park CK, et al: The role of adjuvant radiotherapy in atypical meningioma. J Neurooncol 115:241-247, 2013

35. Pearson BE, Markert JM, Fisher WS, Guthrie BL, Fiveash JB, Palmer CA, et al: Hitting a moving target: evolution of a treatment paradigm for atypical meningiomas amid changing diagnostic criteria. Neurosurg Focus 24(5):E3, 2008

36. Peele KA, Kennerdell JS, Maroon JC, Kalnicki S, Kazim M, Gardner T, et al: The role of postoperative irradiation in the management of sphenoid wing meningiomas. A preliminary report. Ophthalmology 103:1761-1767, 1996

37. Perry A: Meningiomas, in McLendon RE, Rosenblum MK, Bigner DD (eds): Russell \& Rubinstein's Pathology of Tumors of the Nervous System, ed 7. London: Hodder Arnold, 2006, pp 427-474

38. Perry A, Scheithauer BW, Stafford SL, Lohse CM, Wollan PC: "Malignancy" in meningiomas: a clinicopathologic study of 116 patients, with grading implications. Cancer 85:20462056, 1999

39. Perry A, Stafford SL, Scheithauer BW, Suman VJ, Lohse CM: Meningioma grading: an analysis of histologic parameters. Am J Surg Pathol 21:1455-1465, 1997

40. Rogers CL, Perry A, Pugh S, Vogelbaum MA, Brachman D, McMillan W, et al: Pathology concordance levels for meningioma classification and grading in NRG Oncology RTOG Trial 0539. Neuro Oncol 18:565-574, 2016

41. Rogers L, Barani I, Chamberlain M, Kaley T, McDermott M, Raizer J, et al: Meningiomas: knowledge base, treatment outcomes, and uncertainties. A RANO review. J Neurosurg 122:4-23, 2015

42. Rogers L, Mehta M: Role of radiation therapy in treating intracranial meningiomas. Neurosurg Focus 23(4):E4, 2007

43. Simpson D: The recurrence of intracranial meningiomas after surgical treatment. J Neurol Neurosurg Psychiatry 20:22-39, 1957

44. Soyuer S, Chang EL, Selek U, Shi W, Maor MH, DeMonte F: Radiotherapy after surgery for benign cerebral meningioma. Radiother Oncol 71:85-90, 2004

45. Stafford SL, Perry A, Suman VJ, Meyer FB, Scheithauer BW, Lohse CM, et al: Primarily resected meningiomas: outcome and prognostic factors in 581 Mayo Clinic patients, 1978 through 1988. Mayo Clin Proc 73:936-942, 1998

46. Taylor BW Jr, Marcus RB Jr, Friedman WA, Ballinger WE Jr, Million RR: The meningioma controversy: postoperative ra- diation therapy. Int J Radiat Oncol Biol Phys 15:299-304, 1988

47. Willis J, Smith C, Ironside JW, Erridge S, Whittle IR, Everington D: The accuracy of meningioma grading: a 10-year retrospective audit. Neuropathol Appl Neurobiol 31:141149,2005

\section{Disclosures}

Dr. Vogelbaum reports patent and royalty interests in Infuseon Therapeutics, Inc., and receiving personal fees from Neuralstem and from Pharmaco-Kinesis, outside the submitted work. Dr. De Groot reports receiving personal fees from Genentech, VBL Therapeutics, Deciphera Therapeutics, Celldex Therapeutics, Foundation Medicine, Monteris Medical, Omniox, and Oxigene, outside the submitted work; grants from Deciphera Therapeutics, Novartis, and Eli Lilly; and stock ownership in Ziopharm Oncology (also spouse's employer). Dr. Knisely reports receiving support for attending scientific meetings from Brainlab AG, Elekta AB, and Cyber Medical Corporation and an honorarium from Brainlab AG. Dr. Mehta reports receiving personal fees from AbbVie, Novelos, Phillips, BMS, Celldex, Roche, Elekta, Novartis, Cavion, Novocure, Pharmacyclics, and Monteris; and grants from Novelos and Novocure, outside the submitted work.

\section{Author Contributions}

Conception and design: Rogers, Vogelbaum, Alleman, Brachman, Mehta. Acquisition of data: Rogers, Vogelbaum, Ashby, Modi, Galvin, Brachman, Jenrette, De Groot, Bovi, Werner-Wasik, Knisely, Mehta. Analysis and interpretation of data: Rogers, Zhang, Vogelbaum, Perry, Alleman, Brachman, Jenrette, De Groot, Bovi, Werner-Wasik, Knisely, Mehta. Drafting the article: Rogers, Zhang, Vogelbaum, Perry, Galvin, Brachman, Knisely, Mehta. Critically revising the article: all authors. Reviewed submitted version of manuscript: all authors. Approved the final version of the manuscript on behalf of all authors: Rogers. Statistical analysis: Zhang.

\section{Supplemental Information}

\section{Previous Presentations}

Portions of this work were previously presented at the 57th Annual Meeting of the American Society of Therapeutic Radiology and Oncology, San Antonio, Texas, October 2015.

\section{Correspondence}

Leland Rogers, Department of Radiation Oncology, Barrow Neurological Institute, St. Joseph's Hospital and Medical Center, 350 W Thomas Rd., Phoenix, AZ 85013.email: neuropub@ barrowneuro.org. 